\title{
Conformational Analysis in Solution of a Chiral Bisoxazoline Molecule: Vibrational Circular Dichroism Spectroscopy and Density Functional Theory Study
}

\author{
A. Aamouche ${ }^{1}$ and P. J. Stephens ${ }^{2}$ \\ ${ }^{1}$ Faculté Polydisciplinaire de Safi, Université Cadi Ayyad, 46000 Safi, Morocco \\ ${ }^{2}$ Department of Chemistry, University of Southern California, Los Angeles, CA 90089-0482, USA
}

Correspondence should be addressed to A. Aamouche, a.aamouche@ucam.ac.ma

Received 26 July 2011; Accepted 30 August 2011

Academic Editor: Jean-Valère Naubron

Copyright () 2011 A. Aamouche and P. J. Stephens. This is an open access article distributed under the Creative Commons Attribution License, which permits unrestricted use, distribution, and reproduction in any medium, provided the original work is properly cited.

\begin{abstract}
The conformations of the chiral bisoxazoline: 2,2'-methylenebis[3a,8a-dihydro-8H-indeno[1,2-d]oxazole] (also named Ind$a B O X)$, have been studied. Density functional theory (DFT) calculations identify four inequivalent stable conformations. Two, I and II have, $\mathrm{C}_{2}$ symmetry; two, III and IV, have $\mathrm{C}_{1}$ symmetry. The electronic energies of I-IV are ordered: I $<$ II $<$ III $<$ IV. The span in energy is $<1.0 \mathrm{kcal} /$ mole. Vibrational unpolarised absorption and circular dichroism spectra have been predicted for the four conformations using DFT. Comparison of population-weighted spectra to experimental spectra of $\mathrm{CHCl}_{3}$ and $\mathrm{CDCl}_{3}$ solutions in mid-IR region strongly supports the DFT predictions of the number, structures, and relative energies of the conformations of IndaBOX. This shows that DFT predicts spectra with a high degree of reliability. We will undoubtedly illustrate the advantage added by vibrational circular dichroism spectroscopy in conformational analysis and in the absolute configuration determination.
\end{abstract}

\section{Introduction}

We report a study of the conformations of a chiral bisoxazoline: 2,2' -methylenebis[3a,8a-dihydro-8H-indeno[1,2d]oxazole], 1, in solution using ab initio vibrational spectroscopy. The molecular structure of $\mathbf{1}$ is represented on Scheme 1.

In combination with metal salts, chiral bisoxazolines catalyse a wide variety of asymmetric transformations, in many cases with high enantioselectivity [1-5]. To date, although the X-ray structures of several metal complexes of chiral bisoxazolines have been reported [6-10], there appear to have been no structural studies of chiral bisoxazolines alone. Here, we report a study of the bisoxazoline, 1, using ab initio density functional theory (DFT) $[11,12]$, in combination with vibrational unpolarised absorption (IR) and vibrational circular dichroism (VCD) [13-16] spectroscopies. 1 is a flexible molecule; internal rotation can occur about the CC1 and CC2 bonds. We seek to establish the structures and relative energies of the stable conformations of $\mathbf{1}$.
DFT has had a major impact on molecular structure calculations. For many years, wavefunction functional theory (WFT; i.e., HF/SCF and MPs) has been the method of choice. The DFT approach expresses ground-state proprieties, such as total energies, equilibrium positions dipole, and magnetic moments, in terms of electronic density and spin density. The need to include the electron correlation in calculations and the discovery of accurate approximations to exchangecorrelation energy density functional raise the DFT popularity. The computational advantage of DFT originates from the fact that the electron density has three spatial coordinates, regardless of the number of electrons in the system. Thus, DFT allows the calculation of proprieties for systems with almost hundred atoms, a feat not generally possible with high-level WFT methods.

For simplicity, in earliest quantum mechanics treatment, nuclear and electronic motions were separated by using Born-Oppenheimer approximation and by assuming harmonic vibrational motion. To calculate vibrational spectra of a system in a fluid phase, harmonic frequencies and relative 
intensities of each transition are predicted. The IR absorption intensities derive from the dependence of molar extinction coefficient $\varepsilon$ on frequencies $v$. However, VCD intensities originate from the variation of the difference $\Delta \varepsilon(v)=\varepsilon_{L}-\varepsilon_{R}$ resulting from the response to the left $\left(\varepsilon_{L}\right)$ and right $\left(\varepsilon_{R}\right)$ polarization. The theory of electromagnetic wave-molecule interactions, throughout a $g \rightarrow k$ vibrational transition, leads to $(1)[16]$ :

$$
\begin{aligned}
\varepsilon(v) & =\frac{8 \pi^{3} N v}{(2.303) 3000 h c} \sum_{g, k} \alpha_{g} D_{g k} L_{g k}, \\
\Delta \varepsilon(v) & =\frac{32 \pi^{3} N v}{(2.303) 3000 h c} \sum_{g, k} \alpha_{g} D_{g k} L_{g k},
\end{aligned}
$$

where $N, h$, and $c$ are the usual constants; $\alpha_{g}$ is the number of molecules in $g$ state; $L_{g k}$ is the normalized Lorentzian function variable on $v$ and molecular excitation frequency $v_{g k}(2)[17]:$

$$
L_{g k}(v)=\frac{1}{\pi} \frac{\gamma_{g k}^{2}}{\left(v-v_{g k}\right)^{2}+\gamma_{g k}^{2}},
$$

where $\gamma_{g k}$ is the half-width at half-height of the transition line. Dipole strengths $D_{g k}$ and rotatory strengths $R_{g k}$ are linked, respectively, to the electric $\mu_{\mathrm{el}}$ and magnetic $\mu_{\mathrm{mag}}$ operators by means of transition moments (3) [18]:

$$
\begin{gathered}
D_{g k}=\left|\left\langle g\left|\vec{\mu}_{\mathrm{el}}\right| k\right\rangle\right|^{2}, \\
R_{g k}=\operatorname{Im}\left\{\left\langle g\left|\vec{\mu}_{\mathrm{el}}\right| k\right\rangle \bullet\left\langle k\left|\vec{\mu}_{\mathrm{mag}}\right| g\right\rangle\right\} .
\end{gathered}
$$

Considering only the vibrational ground state $g=0$ and fundamental transitions involving one mode alone to the states $k=1$, the fundamental transition moments in mode $i$ are (4):

$$
\begin{gathered}
\left\langle 0\left|\left(\mu_{\mathrm{el}}\right)_{\beta}\right| 1\right\rangle_{i}=\left(\frac{\hbar}{4 \pi \omega_{i}}\right)^{1 / 2} \sum_{\lambda \alpha} S_{\lambda \alpha, i} P_{\alpha \beta}^{\lambda}, \\
\left\langle 0\left|\left(\mu_{\mathrm{mag}}\right)_{\beta}\right| 1\right\rangle_{i}=-\left(4 \pi \hbar^{3}\right)^{1 / 2} \sum_{\lambda \alpha} S_{\lambda \alpha, i} M_{\alpha \beta}^{\lambda},
\end{gathered}
$$

where $P_{\alpha \beta}^{\lambda}$ are the atomic polar tensors (APTs) and $M_{\alpha \beta}^{\lambda}$ are the atomic axial tensors (AATs) for the nucleus $\lambda$ along the directions $\alpha, \beta=x, y$, or $z$. $S_{\lambda \alpha, i}$ are the transformation matrix elements between nuclear Cartesian coordinates $X$ and nuclear normal coordinates $Q$ (5) [19]:

$$
X_{\lambda \alpha}=\sum_{i} S_{\lambda \alpha, i} Q_{i}
$$

Basically to get all possible conformations for any molecule, the potential energy surface (PES) needs first to be scanned. After the resolution of all optimal molecular geometries, the harmonic force field (HFF), the energies, and the electronic population are obtained. The diagonalization of the Hessian matrix (HFF with respect to nuclear Cartesian coordinates $X$ ) produces normal modes frequencies, the

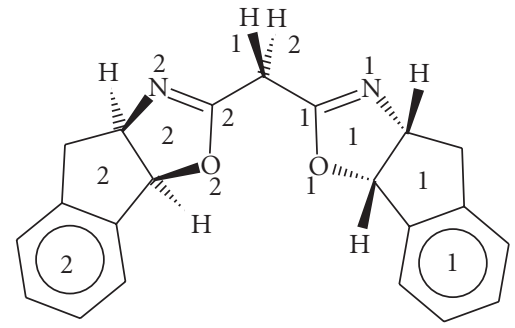

(+)-1

Scheme 1

vibronic wavefunctions, and the transformation matrix $S$. In sum, the prediction of the IR and VCD spectra requires the calculation of three major parameters: (i) the fundamental frequencies $v_{i}$; (ii) the dipole strengths $D_{i}$ (via harmonic APTs elements) for the IR lines; (iii) the rotatory strengths $R_{i}$ (via APTs and AATs elements) for the VCD peaks.

In practice, the AATs elements cannot be determined within Born-Oppenheimer approximation. This occurs from the vanishing of the electronic contribution to the magnetic moment $\mu_{\mathrm{mag}}$ associated with the vibrational transition in the ground electronic state $[20,21]$. So the exact formulations of VCD intensities has been resolved by taking into account high-order corrections in vibronic wavefunctions. The admixture of electronic excited state and ground states wavefunctions was accomplished by using nuclear kinetic energy or magnetic field perturbation theories [21, 22].

Over the last decades, DFT has increasingly become the method of choice in predicting molecular properties. The variety of properties which can be calculated using DFT has increased dramatically. Subsequently, the functionals sophistication and accuracy has increased; particularly important has been the development of hybrid density functionals. Becke had constructed new functionals (B3) taking into consideration the exchange and the correlation aspects. Additional suggestions gives birth to the most popular functionals B3LYP (Lee, Yang, and Parr) and B3PW91 (Perdew and Wang) [23-25]. These functionals describe correctly the electronic proprieties of numerous molecular and materials compounds.

Elsewhere to describe molecular orbitals, many basis sets are applied. They are a linear combination of a series of atomic orbital exponentials, usually Gaussian functions [26]. In our calculation the choice of the basis set $6-31 \mathrm{G}^{*}$ and of the functional (B3PW91) has enabled an optimal exploitation of the computational resources via Gaussian program and deliver remarkably precise results for various systems [27]. Of particular interest here is the development of analytical derivative methods for calculating harmonic force fields (HFFs) and atomic polar tensors (APTs), which together permit the prediction of vibrational unpolarised absorption spectra, and for calculating atomic axial tensors (AATs), which together with HFFs and APTs permit the prediction of VCD spectra for chiral molecules [16]. As a result of the use of Gauge-invariant atomic orbitals (GIAOs), AATs are calculated at the same level of accuracy as APTs. 
Absorption and VCD spectra were synthesized from the calculated frequencies, the dipole strengths, and the rotatory strengths, assuming constant Lorentzian bandshapes $(\gamma=$ $4 \mathrm{~cm}^{-1}$ ). Intrinsically, the VCD sign and magnitude are expressed by the anisotropy ratio $g$. This ratio is defined experimentally as the VCD extinction coefficient $\Delta \varepsilon$ to the IR extinction coefficient $\varepsilon ; g=\Delta \varepsilon / \varepsilon$. Theoretically, $g$ is linked to the quotient of the rotatory strength $R$ and the dipole strength $D ; g=4 R / D$. This leads to VCD signals normally four to six orders of magnitude smaller than the IR signals.

Studies of a number of rigid chiral molecules have shown that DFT, implemented using hybrid functionals and sufficiently large basis sets, can predict absorption and VCD spectra with a high degree of reliability [28-35]. As a result, DFT, in combination with absorption and VCD spectroscopies, provides a reliable basis for analyzing the conformational structures of flexible chiral molecules. A number of such molecules have already been studied [36-45]. Here, we extend these studies to the chiral bisoxazoline, 1 (known as IndaBOX). The involvement of this compound in many asymmetric synthesis, catalysis, and surface grafting imposes the structural characterization including its chirality. DFT calculation on this type of ligands was first performed for isobutyleneBox [46]. This structurally simple $\mathrm{C}_{2}$ symmetry case was compared with NMR spectroscopic parameters to probe the behavior as chelate complex with $\mathrm{Cu}(\mathrm{I})$. The configuration of $\mathbf{1}$ analogs was extensively investigated in many references [47-53]. The present VCD study is original on such chiral molecules. We will report here the structural parameters of 1 determined by DFT calculation along with the corresponding IR and VCD spectra compared to the measurements.

\section{Methods}

Experimental unpolarised vibrational absorption spectra of 1 were obtained in solution in $\mathrm{CHCl}_{3}, \mathrm{CDCl}_{3}$, and $\mathrm{CH}_{2} \mathrm{Cl}_{2}$ using a Nicolet MX1 spectrometer at a resolution of $1 \mathrm{~cm}^{-1}$. Experimental VCD spectra were obtained using a Bomem/BioTools ChiralIR spectrometer at a resolution of $4 \mathrm{~cm}^{-1}$. VCD spectra of $(+)$ - and $(-)$-1 were obtained using scan times of 1 hour. The VCD spectrum of $( \pm)$ 1 constituted the baseline. The "half-difference" spectrum, $[\Delta \varepsilon(-)-\Delta \varepsilon(+)] / 2$, and the "half-sum" spectrum, $[\Delta \varepsilon(-)+$ $\Delta \varepsilon(+)] / 2$, were obtained thence. The former provides the final VCD spectrum. The latter defines the reliability of the VCD spectrum; deviations from zero are due to noise and to polarization artifacts which are not identical in the $(+)$, $(-)$, and $( \pm)$ spectra. All experiments used a variable-path cell (Specac) with $\mathrm{KBr}$ windows.

$(+)$ - and $(-)-1$ were obtained from Aldrich. Specified chemical purities were $98 \%$ for both $(+)$ - and (-)-1. Specified specific rotations $[\alpha]_{D}^{22}$ were +353 and $-353^{\circ}(c=$ 3.7, $\left.\mathrm{CHCl}_{3}\right)$, respectively. Solutions of $( \pm)-1$ were obtained by mixing solutions of $(+)$ - and $(-)-1$.

Hartree-Fock (HF) and DFT calculations of (+)-1 were carried out using Gaussian [27]. The functionals used in DFT calculations were B3PW91 and B3LYP. The 6-31G* and STO-3G basis sets were used. Unpolarised absorption and VCD spectra were synthesized from frequencies, dipole strengths, and rotational strengths, assuming Lorentzian band shapes.

Studies on rigid molecules had proven their exactness mostly for the absolute configuration determination as the conformation of such molecules is unique and stable. However, flexible molecules require conformations search for instance by scanning freely the torsional bonds. Therefore, the torsions multiplicity complicates definitely the potential energy surface (PES), exploration. Once the structure and relative energy of each stable conformation is established, the subsequent vibrational spectra are calculated. Assuming a Boltzmann distribution, the composite theoretical spectra are summed, weighting each by the fractional population, giving conformationally averaged spectra. Vibrational spectra are an exquisitely sensitive function of molecular structure. In particular, in the case of flexible molecules, vibrational spectra depend sensitively on conformation and provide a basis for conformational analysis. In the case of chiral molecules, VCD spectra are generally more sensitive to conformation than absorption spectra. As a result, in the conformational analysis of chiral molecules the combined use of absorption and VCD spectra is substantially more powerful than the use of absorption spectra alone. The association of both IR and VCD (predicted and experimental) spectra yields more credible assignment as the bands are now either positive or negative.

\section{Results}

Internal rotation in $\mathbf{1}$ can occur about the $\mathrm{C}-\mathrm{C} 1$ and $\mathrm{C}-\mathrm{C} 2$ bonds. We have searched for stable conformations as follows. First, an initial exploration at the HF/STO-3G level located a stable conformation possessing $\mathrm{C}_{2}$ symmetry: structure $\mathrm{I}$. This structure was reoptimized at the B3PW91/6-31G* level. Next, a scan of the PES, varying the two dihedral angles $\phi_{1}$ $=\mathrm{H} 1 \mathrm{CC} 1 \mathrm{O} 1$ and $\phi_{2}=\mathrm{H} 1 \mathrm{CC} 2 \mathrm{O} 2$ independently, was carried out over the ranges $\phi_{1}, \phi_{2}=-180-+180^{\circ}$, in steps of $10^{\circ}$. All other internal coordinates were fixed at the values of the B3PW91/6-31G* optimized structure. The scan was carried out at the HF/STO-3G level. The results obtained are shown in Figure 1. The PES exhibits six minima. One corresponds to structure I. The five additional minima are labelled II, IIIa, IIIb, IVa, and IVb. Structures II, IIIa, IIIb, Iva, and IVb were then optimised at the B3PW91/6-31G* level. Structure II possesses $\mathrm{C}_{2}$ symmetry. Structures IIIa, IIIb, IVa, and IVb possess $C_{1}$ symmetry. IIIa and IIIb are equivalent, as are IVa and IVb. IIIa and IIIb are interconverted by rotation of the molecule by $180^{\circ}$; IVa and IVb are interconverted in the same way.

The four inequivalent B3PW91/6-31G* structure are shown in Figure 2. The main structural parameters of the (O1C1N1)CH1H2(O2C2N2) moieties are given in Table 1. The obtained electronic energies of I-IV are given in Table 2. The span is $<1 \mathrm{kcal} / \mathrm{mole}$, and the order is I $<$ II $<$ III $<$ IV. Populations predicted from the B3PW91 and B3LYP energies are also given in Table 2. Structures I-IV have also 
Table 1: Nonbonded distances $(\AA)$ in the $(\mathrm{O} 1 \mathrm{C} 1 \mathrm{~N} 1) \mathrm{CH} 1 \mathrm{H} 2(\mathrm{O} 2 \mathrm{C} 2 \mathrm{~N} 2)$ moieties for the four conformations of $(+)-1$.

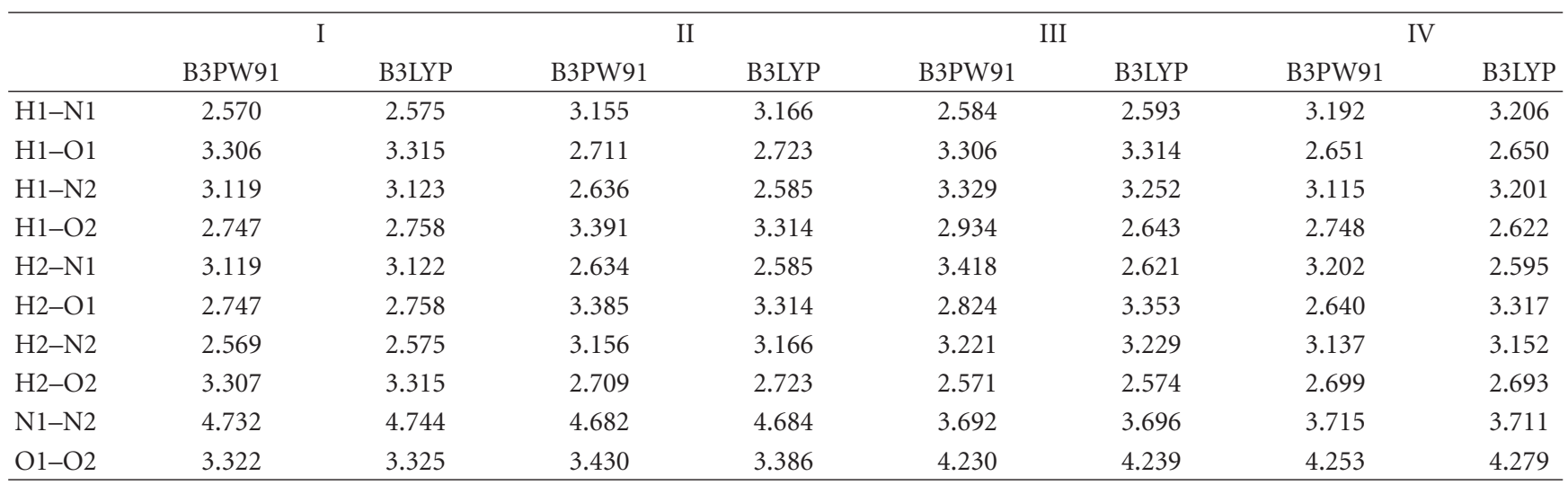

TABLE 2: Electronic energies E and populations $\mathrm{P}$ of conformations I, II, III, and IV of $\mathbf{1}^{\mathrm{a}}$.

\begin{tabular}{lcccc}
\hline & \multicolumn{2}{c}{ B3PW91/6-31G* } & \multicolumn{2}{c}{ B3LYP/6-31G* } \\
& $\mathrm{E}$ & $\mathrm{P}$ & $\mathrm{E}$ & $\mathrm{P}$ \\
\hline I & 0.00 & 49.2 & 0.00 & 48.0 \\
II & 0.41 & 24.8 & 0.33 & 27.5 \\
III & 0.71 & 15.0 & 0.77 & 13.1 \\
IV & 0.89 & 11.0 & 0.85 & 11.4 \\
\hline
\end{tabular}

${ }^{\mathrm{a}} \mathrm{E}$ in $\mathrm{kcal} / \mathrm{mol}, \mathrm{P}$ in \% (calculated assuming a Boltzmann distribution and $\left.T=293^{\circ} \mathrm{K}\right)$.

been optimized at the B3LYP/6-31G* level. Qualitatively, the structures are identical to the B3PW91/6-31G* structures. Quantitative differences in bond lengths, bond angles, and dihedral angles are small. B3LYP/6-31G* and B3PW91/6$31 \mathrm{G}^{*}$ energies differ by $<0.1 \mathrm{kcal} / \mathrm{mole}$. Once we take into account the free thermal energy, the B3PW91/6-31G* energy differences decreases. The free energies of I-IV are differently ordered: I $<$ III $<$ IV $<$ II. More spectacularly, the span in free energies is smaller $<0.3 \mathrm{kcal} / \mathrm{mole}$ (III versus I drop to $0.05 \mathrm{kcal} / \mathrm{mol}$, IV versus I becomes $0.19 \mathrm{kcal} / \mathrm{mol}$, whereas II versus $\mathrm{I}$ is $0.26 \mathrm{kcal} / \mathrm{mol}$ ). The validity of stability lowering for the conformation II should be estimated with a tightest spectral analysis.

In order to assess the reliability of these predictions we have compared the vibrational absorption and circular dichroism spectra predicted for structures I, II, III, and IV, and for mixtures of these structures, to the experimental spectra. See Table 3, placed in a supplementary information section available online at doi:10.1155/2011/905045, that shows the whole 123 B3PW91/6-31G* vibrational modes listing.

\section{Discussion}

Our DFT calculations show that (1) several stable conformations of 1 exist, differing very little $(<1 \mathrm{kcal} / \mathrm{mole})$ in energy; (2) in each conformation, the CNO plane of each oxazole ring is oriented in one of three orientations, interrelated by $\sim 120^{\circ}$ rotations about the CC1 or CC2 bonds; (3) of the possible combinations of $\mathrm{C} 1 \mathrm{~N} 1 \mathrm{O} 1$ and $\mathrm{C} 2 \mathrm{~N} 2 \mathrm{O} 2$ orientations, four correspond to inequivalent stable

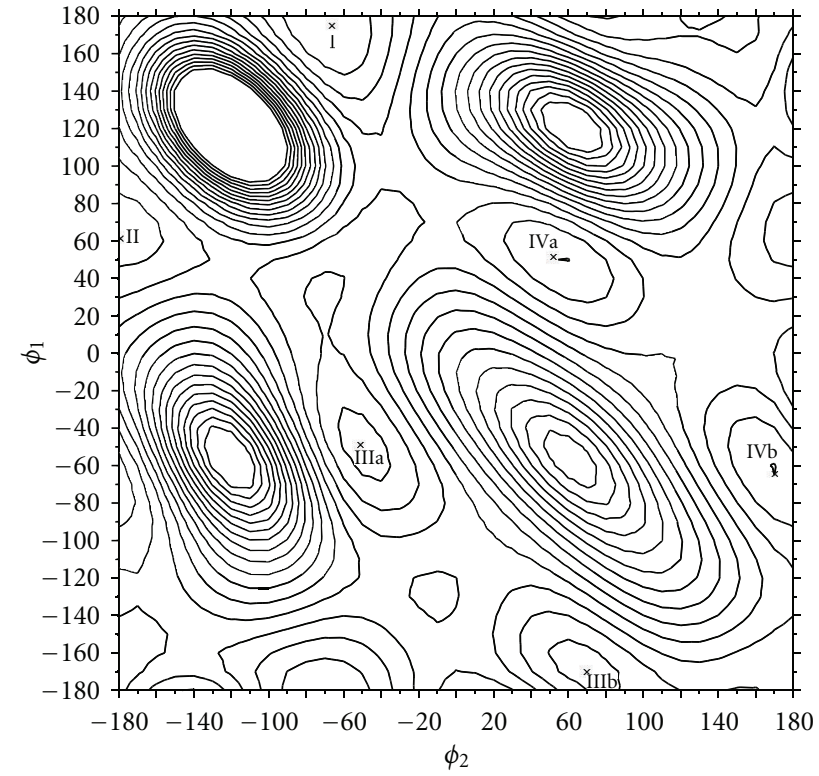

Figure 1: HF/STO-3G PES of 1. $\phi_{1}=\mathrm{H} 1 \mathrm{CC} 1 \mathrm{O} 1$ and $\phi_{2}=$ $\mathrm{H} 1 \mathrm{CC} 2 \mathrm{O} 2$. All other internal coordinates are fixed at the B3PW91/6-31G* optimized values of structure I. Contour spacing is $0.5 \mathrm{kcal} / \mathrm{mole}$. The values of $\phi_{1}, \phi_{2}$ for structures I, II, IIIa, IIIb, $\mathrm{IVa}$, and IVb after optimization at the B3PW91/6-31G* level are indicated $(\mathrm{x})$.

conformations; (4) the two lowest energy conformations, I and II, possess $\mathrm{C}_{2}$ symmetry; the two of highest energy, III and IV, possess $\mathrm{C}_{1}$ symmetry.

To shed light on the results obtained for $\mathbf{1}$, we have studied the molecule methyl oxazole, 2 . Its molecular structure is represented on Scheme 2.

Specifically, we have calculated the PES of 2 with respect to rotation of the methyl group at the B3PW91/6-31G* level. Minima are found at $120^{\circ}$ intervals. In each stable conformation, one $\mathrm{C}-\mathrm{H}$ bond of the methyl group is coplanar with the NCO moiety of the oxazole ring; the C$\mathrm{H} \mathrm{H}$ is "cis" with respect to the NCO N and "trans" with respect to the NCO O. The barrier to internal rotation is $1.3 \mathrm{kcal} / \mathrm{mole}$; at the top of the barrier, one $\mathrm{C}-\mathrm{H}$ bond is coplaner with the NCO group, its $\mathrm{H}$ being "cis" to O and 


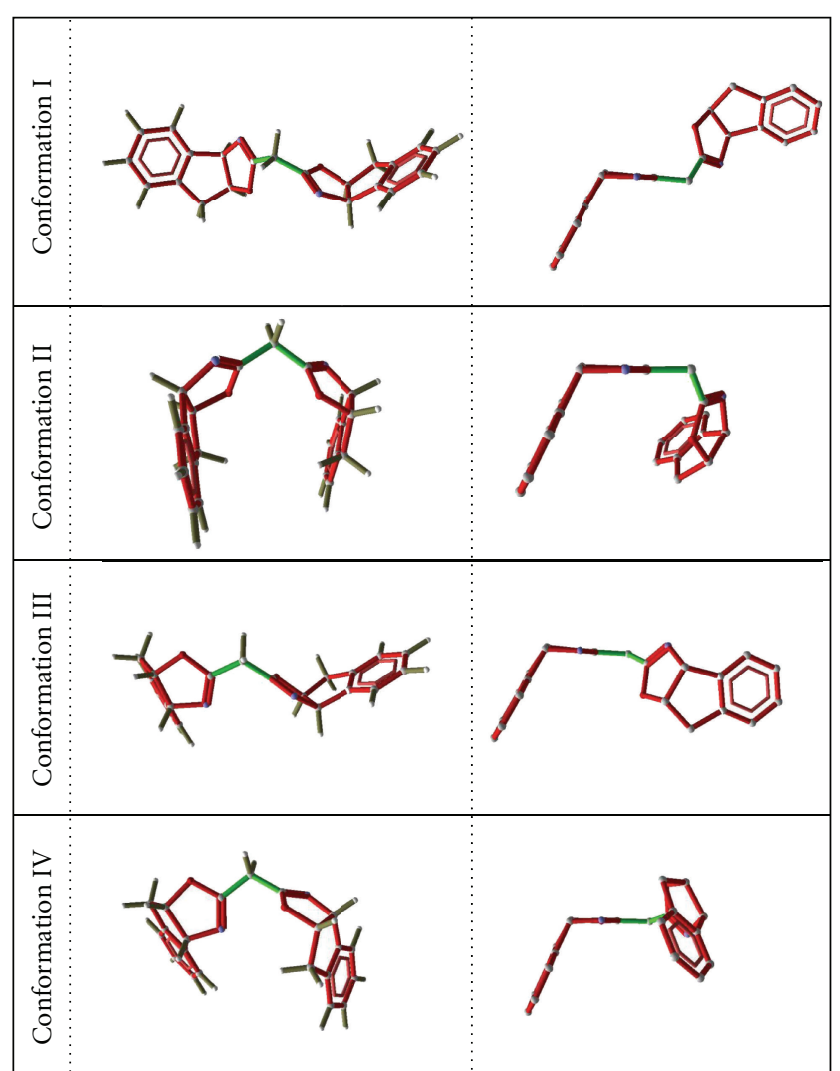

FIGURE 2: The B3PW91/6-31G* structure of 1. Each one of the four conformations is visualized from two orientations. On the left side is an optimal view (including hydrogens), whereas the right side view gives one moiety versus the plan of the other.

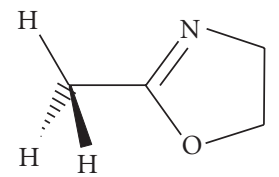

2

SCHeme 2

"trans" to N. Thus, the alignment of the NCO groups in the most stable conformation I is reasonable.

In structure I, the $\mathrm{N} 1 \mathrm{C} 1 \mathrm{O} 1$ and $\mathrm{N} 2 \mathrm{C} 2 \mathrm{O} 2$ planes are very nearly coplanar with the $\mathrm{CH} 1$ and $\mathrm{CH} 2$ bonds, respectively. $\mathrm{N} 1$ and $\mathrm{N} 2$ and $\mathrm{O} 1$ and $\mathrm{O} 2$ are symmetrically located with respect to the $\mathrm{C} 1 \mathrm{CC} 2$ plane. In structure II, the N1C1O1 and $\mathrm{N} 2 \mathrm{C} 2 \mathrm{O} 2$ planes are very nearly coplanar with the $\mathrm{CH} 2$ and $\mathrm{CH} 1$ bonds, respectively. Again, $\mathrm{N} 1$ and N2 and $\mathrm{O} 1$ and $\mathrm{O} 2$ are symmetrically located with respect to the C1CC2 plane. Thus, structure II is obtained from structure I by a rotation of each NCO group by $\sim 120^{\circ}$. Qualitatively, the $(\mathrm{N} 1 \mathrm{C} 1 \mathrm{O} 1) \mathrm{CH} 1 \mathrm{H} 2(\mathrm{~N} 2 \mathrm{C} 2 \mathrm{O} 2)$ moiety of structure II is obtained by reflection of that of structure I in the C1CC2 plane. In structures III and IV, the orientations of the $\mathrm{O} 1 \mathrm{C} 1 \mathrm{~N} 1$ and $\mathrm{CH} 1 \mathrm{H} 2$ groups are qualitatively the same as in structures I and II, respectively. The $\mathrm{O} 2 \mathrm{C} 2 \mathrm{~N} 2$ groups, on the other hand, are oriented differently. In both structures III and $\mathrm{IV}$, the $\mathrm{O} 2 \mathrm{C} 2 \mathrm{~N} 2$ plane nearly bisects the $\mathrm{CH} 1 \mathrm{H} 2$ angle, with $\mathrm{N} 2$ nearly eclipsing $\mathrm{C} 1$.

The structures of the three rings of the oxazilidine moieties of 1 vary very little with conformation. All are close to planar. The maximum dihedral angle for the five atoms of the oxazole ring is $7.0^{\circ}$ in I, $6.6^{\circ}$ in II, $8.8^{\circ} / 6.9^{\circ}$ in III, and $9.3^{\circ} / 8.0^{\circ}$ in IV (where the rings are inequivalent, the two angles are for rings $1 / 2$ ). The maximum dihedral angle for the five atoms of the cyclopentane ring is $7.3^{\circ}$ in I, $7.3^{\circ}$ in II, $9.1^{\circ} / 7.3^{\circ}$ in III, and $10.0^{\circ} / 8.6^{\circ}$ in IV. The maximum dihedral angle of the six atoms of the benzene ring is $0.3^{\circ}$ in $\mathrm{I}, 0.3^{\circ}$ in II, $0.3^{\circ} / 0.3^{\circ}$ in III, and $0.6^{\circ} / 0.5^{\circ}$ in IV.

We have evaluated the structures and relative energies of the conformations of $\mathbf{1}$ predicted by DFT using vibrational spectroscopy. Calculated vibrational unpolarised absorption and VCD spectra of conformations I, II, III, and IV differ significantly, the variation being dramatically greater for the VCD spectra. As a result, the predicted spectra of 1 are sensitive to the conformational composition. Using populations calculated assuming that the conformational free energy differences are equal to the DFT energy differences, we have synthesized composite spectra for 1 and compared them to the experimental spectra in the mid-IR spectral region. Allowing for the limitations of B3PW91 and B3LYP harmonic calculations at the $6-31 \mathrm{G}^{*}$ basis set level, the results are in convincing agreement with experiment.

Experimental mid-IR absorption and VCD spectra of 1, measured in $\mathrm{CHCl}_{3}$ and $\mathrm{CDCl}_{3}$, are shown in Figure 3. Mid-IR absorption and VCD spectra predicted for I-IV at the B3PW91/6-31G* level are shown in Figures 4, 5, 6, 7,8 , and 9 , together with the composite spectra obtained by summing the population-weighted contributions of I-IV and the experimental spectra. Examination of Figures 4-9 leads to the following broad conclusions:

(1) the predicted spectra of I, II, III, and IV differ; the VCD spectra vary much more dramatically than the absorption spectra;

(2) the contributions of I to the predicted composite absorption and VCD spectra predominate, due to its greater population;

(3) the contributions of II, III, and IV are quantitatively significant in certain spectral regions; as a result, the predicted composite spectra differ significantly from the spectra of I alone. The difference is greater for the VCD spectra than for the absorption spectra;

(4) several modes of II, III, and/or IV are predicted to give rise to absorption and/or VCD resolved from the modes of I;

(5) the composite absorption and VCD spectra are in good overall agreement with the experimental spectra; the contributions of II, III, and IV are clearly discernible.

In order to compare the predicted and experimental spectra in close detail, we have divided the whole spectrum into many regions. Here, we choose to point the most significant features and changes. 
The first noteworthy section is located in Figures 4 and 5 (between $800 \mathrm{~cm}^{-1}$ and $1150 \mathrm{~cm}^{-1}$ ). The absorption "A," containing several, partially resolved bands, can be assigned predominantly to modes $40-45$ of I, modes $40-45$ of II, and modes 44 and 45 of III and IV. The agreement of predicted and experimental spectra is poor, and detailed assignment is not straightforward. The VCD " $\mathrm{A}$ " is oscillatory, with two + features corresponding to the two absorption maxima and two features in between and to higher frequency of these features, respectively. The predicted VCD exhibits the same qualitative behavior. The lower frequency +, features originate predominantly in modes 40/41 and 42 of $\mathrm{I}$, respectively. The higher frequency,+- features are dominated by modes 44 and 45 of II respectively. (Note that modes 44 and 45 of I are predicted to give - and + VCD, respectively, opposite in sign to the observed signs of the two higher frequency features.) The absorption " $\mathrm{B}$ " can be assigned predominantly to modes 46 of I and 47 of II and III. The VCD " $\mathrm{B}$ " is + , in contrast to the predicted VCD, predominantly due to modes $46 / 47$ of I and 47 of II, which are - . The absorption " $\mathrm{C}$ " is composite, with a clearly resolved shoulder to lower frequency of the peak. The two features are assigned predominantly to modes 49 and 50 of I. The VCD " $\mathrm{C}$ " is +; a shoulder to lower frequency of the peak is barely visible. The predicted VCD is + , originating predominantly in modes 50 of I and II, with a weak + feature at lower frequency originating predominantly in modes 49 of II and III. (Note that both modes 48 and 49 of I are predicted to give weak VCD.) The absorption " $D$ " can be assigned predominantly to modes $51 / 52$ of I and II. The VCD of "D" is -, in agreement with the predicted VCD, which originates predominantly in modes 52 of I and II. The absorption "E" can be assigned to modes 54 of I and II. The VCD "E" is large and +, as is the predicted VCD, which originates predominantly in mode 54 of I. The absorption "F" consists of a very strong central feature, with a shoulder clearly resolved to lower frequency and several shoulders visible to higher frequency. The predicted absorption consists of a strong central feature, attributable predominantly to modes $58-60$ of I, mode 58 of II, modes $57 / 58$ of III, and mode 58 of IV; a strong shoulder to lower frequency, originating in modes 57 of I, II, and IV; a weak shoulder to higher frequency originating in modes 63 of I and II. We assign the latter to the highest frequency shoulder observed experimentally. The observed VCD " $F$ " is large and bisignate, as in the predicted VCD, which originates predominantly in modes 58-60 of I for the - band and in mode 63 of I and II for the + band (note that the later mode changes the sign between the two conformation). The absorption " $G$ " can be assigned to modes 64 of I, II, and IV. The VCD " $G$ " is and very weak. The predicted VCD is also very weak, but +, originating predominantly in mode 64 of II. The absorption " $\mathrm{H}$ " is assignable to modes $65 / 66$ of I, II, III, and IV. The VCD " $\mathrm{H}$ " is + , consistent with the predicted VCD, which originates predominantly in modes 65 of I, 66 of II, 66 of III, and 65/66 of IV.

The second notable section is located in Figures 6 and 7 (between $1100 \mathrm{~cm}^{-1}$ and $1550 \mathrm{~cm}^{-1}$ ). The absorption

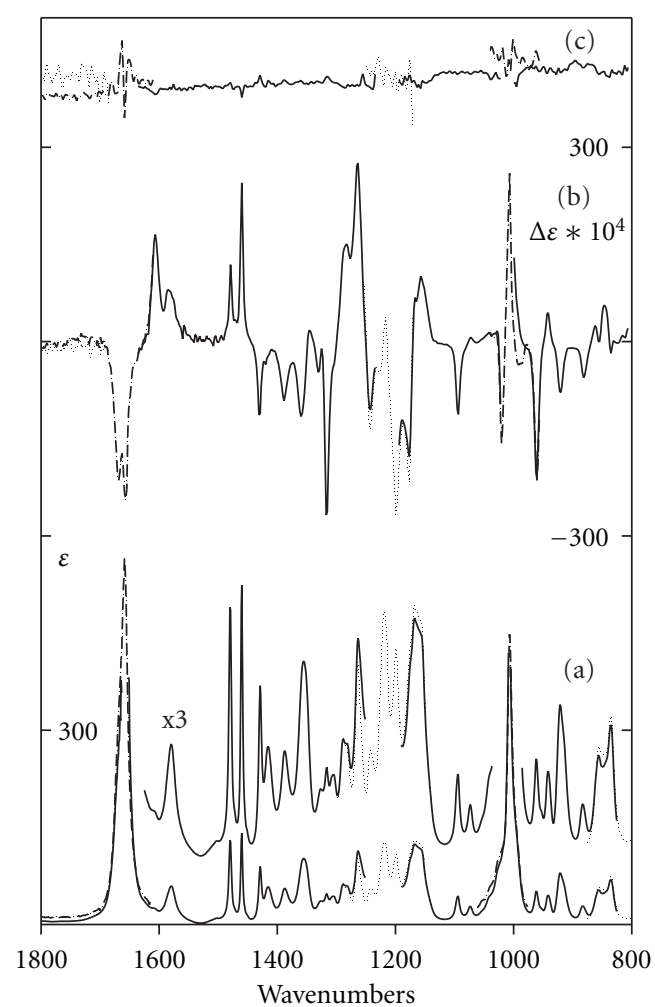

FIGURE 3: Mid-IR $\left(800-1800 \mathrm{~cm}^{-1}\right)$ absorption and VCD spectra of 1. - : IR: (825-1005, 1008-1190, 1250-1652, 1663-1800 cm-1), VCD: $\left(806-1000,1022-1194,1234-1636 \mathrm{~cm}^{-1}\right), 0.50 \mathrm{M}$ in $\mathrm{CHCl}_{3}$; dots line: IR: $\left(800-875,1131-1300 \mathrm{~cm}^{-1}\right)$, VCD: $1171-1254 \mathrm{~cm}^{-1}$, $0.40 \mathrm{M}$ in $\mathrm{CDCl}_{3}$; dashed line: IR: $\left(976-1061,1615-1800 \mathrm{~cm}^{-1}\right)$, VCD: (957-1040, 1610-1800 cm ${ }^{-1}$ ), 0.20M in $\mathrm{CH}_{2} \mathrm{Cl}_{2}$. (a) Absorption spectrum, (b) VCD "half-difference" spectrum of (+)-1, (c) VCD "half-sum" spectrum.

"J" contains several partially resolved bands. The predicted absorption is also complex, originating in modes $67-73$ of I and II and 67-74 of III and IV. The VCD "J" is bisignate. The VCD can be assigned predominantly to modes 67-71 of I; the + VCD originates principally in modes 73 of I, modes $72 / 73$ of II, and modes 73 of III and IV. The absorption "K" originates predominantly in modes $74 / 75$ of I and II. The VCD " $\mathrm{K}$ " is large and +. The predicted VCD is +, originating predominantly in modes 74/75 of I and 74 of II, but relatively weak. The absorption "L" can be assigned predominantly to modes 78 of I and II. The VCD "L" is -, consistent with the predicted VCD, which originates predominantly in modes 78 of I and II. (Note that the VCD of II is opposite in sign to that of I and the predicted VCD involves a large cancellation of oppositely signed contributions.) The weak absorption " $\mathrm{M}$ " is attributed predominantly to mode 79 of I. The VCD " $M$ " is strong and +, consistent with the predicted VCD which originates predominantly in mode 79 of I. The absorption "N" consists of a peak together with a shoulder to lower frequency. The former can be assigned to mode 80 of I; the latter can be assigned to modes 80 of II, III, and IV. The VCD " $\mathrm{N}$ " is - and strong; VCD corresponding to the low frequency shoulder is not clearly observed. The predicted VCD of modes 80 of I-IV is uniformly -, that of I 


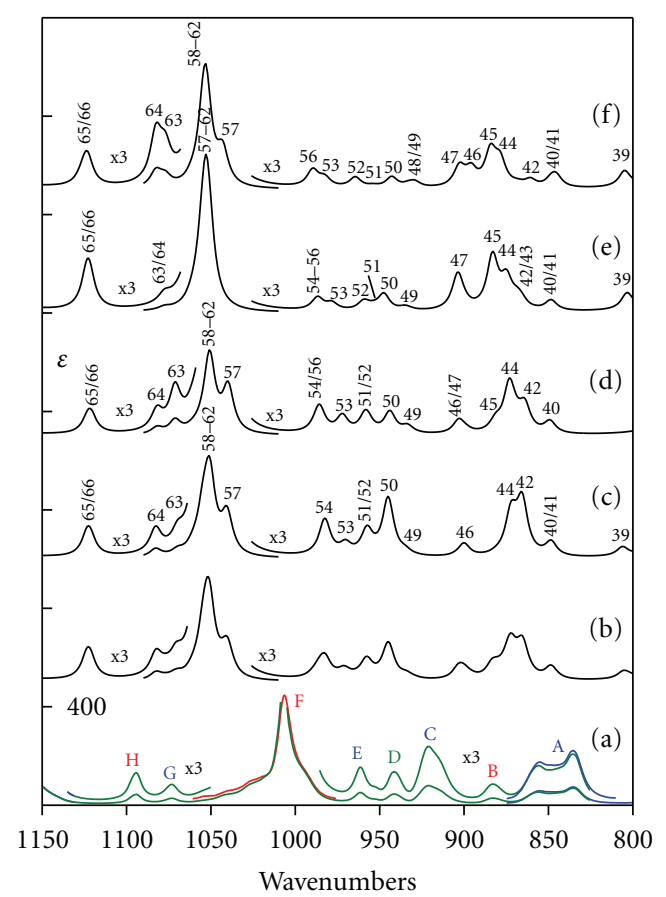

FIgURE 4: Mid-IR (800-1150 $\mathrm{cm}^{-1}$ ) absorption spectrum of 1. (a) Experimental spectrum (from Figure 3); (b) composite calculated spectrum; (c) B3PW91/6-31G* spectrum of I; (d) B3PW91/6-31G* spectrum of II; (e) B3PW91/6-31G* spectrum of III; (f) B3PW91/6$31 \mathrm{G}^{*}$ spectrum of IV. The composite spectrum is obtained using the percentage populations of I-IV in Table 2. Fundamentals are numbered. Band shapes are Lorentzian $\left(\gamma=4.0 \mathrm{~cm}^{-1}\right)$.

being strong, in agreement with experiment. The absorption "P" comprises two, partially resolved bands. The lower frequency component is assignable predominantly to mode 83 of II, while the higher frequency component is assignable predominantly to modes 85 of I and II. The VCD "P" is unresolved and -. The absorption "Q" comprises a triplet of bands, assignable predominantly to mode 86 of I, modes $87 / 88$ of I and II, and modes 88 of III and IV, respectively. The VCD "Q" is strong and + in the central feature and weaker and + in the two outer features. The strong VCD originates predominantly in mode 87 of I. The lower frequency feature is assignable to mode 86 of I and mode 87 of IV. The higher frequency feature is assignable to mode 88 of II. The absorption " $R$ " is broad and attributable to modes 89-92 of I and II, together with mode 90 of IV. The predicted VCD is bisignate: the lower frequency component originates predominantly in modes 89 of I and 90 of II; the higher frequency + component originates predominantly in modes 91/92 of I and II (note that the contributions of I and II are of opposite sign). The predicted VCD is in qualitative agreement with the bisignate VCD observed experimentally. The absorption " $\mathrm{S}$ " originates exclusively in modes 92 of III and IV; no absorption of I or II is predicted in this region. The VCD of both modes are predicted to be + , in agreement with the observed VCD. The absorption " $T$ " is assignable to modes 93 of I, II, III, and IV. The VCD "T" is weak and + . The predicted VCD is also + and weak,

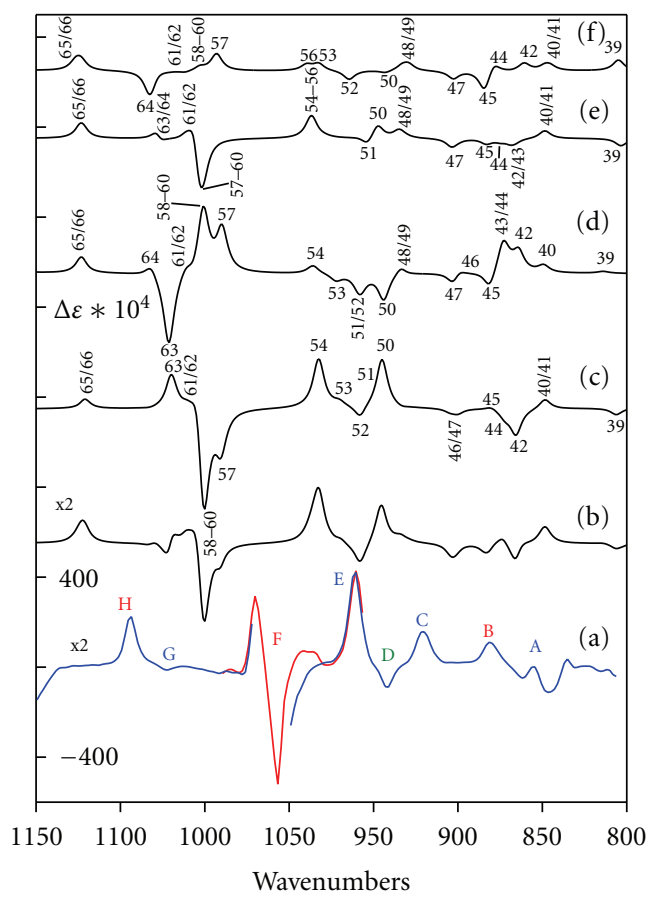

Figure 5: Mid-IR $\left(800-1150 \mathrm{~cm}^{-1}\right)$ VCD spectrum of 1. (a) Experimental spectrum (from Figure 3); (b) composite calculated spectrum; (c) B3PW91/6-31G* spectrum of I; (d) B3PW91/6-31G* spectrum of II; (e) B3PW91/6-31G* spectrum of III; (f) B3PW91/6$31 \mathrm{G}^{*}$ spectrum of IV. The composite spectrum is obtained using the percentage populations of I-IV in Table 2. Fundamentals are numbered. Band shapes are Lorentzian $\left(\gamma=4.0 \mathrm{~cm}^{-1}\right)$.

originating predominantly in I, II, and III. The absorption "X" is assignable to modes $94 / 95$ of I, II, III, and IV. The VCD "X" is +. The predicted VCD is also +, originating in all four conformations. The absorption "Y" is assignable to modes 96/97 of I, II, III and IV. The VCD "Y" is -. The predicted VCD is also -, originating in all four conformations. The absorption " $\mathrm{Z}$ " is assignable to modes $98 / 99$ of I, II, III and IV. The VCD " $\mathrm{Z}$ " is - . The predicted VCD is also -, originating in all four conformations.

Above $1500 \mathrm{~cm}^{-1}$ (Figures 8 and 9), three absorption

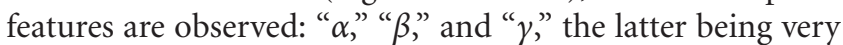
intense. The absorptions " $\alpha$ " and " $\beta$ " are assignable predominately to modes $100 / 101$ and $102 / 103$ of I, respectively. The absorption " $y$ " exhibits a shoulder to higher frequency of the peak. The peak absorption is assigned to modes 104 of I and II. The shoulder is assigned to modes $104 / 105$ of III and IV. The VCD " $\alpha$ " and " $\beta$ " are - in agreement with the predicted VCD which is attributable to modes 100/101 and 102/103 of I, II, III, and IV, respectively. The VCD " $\gamma$ " comprises two + features. The lower frequency component is assignable to modes 104 of I and II; the higher frequency component is attributable predominantly to mode 105 of I.

Ultimately, the calculated composite absorption and VCD spectra thus yield a satisfactory assignment of the experimental absorption and VCD spectra. Unambiguous evidence of significant populations of II, III, and IV is provided by the detailed comparison of predicted and 


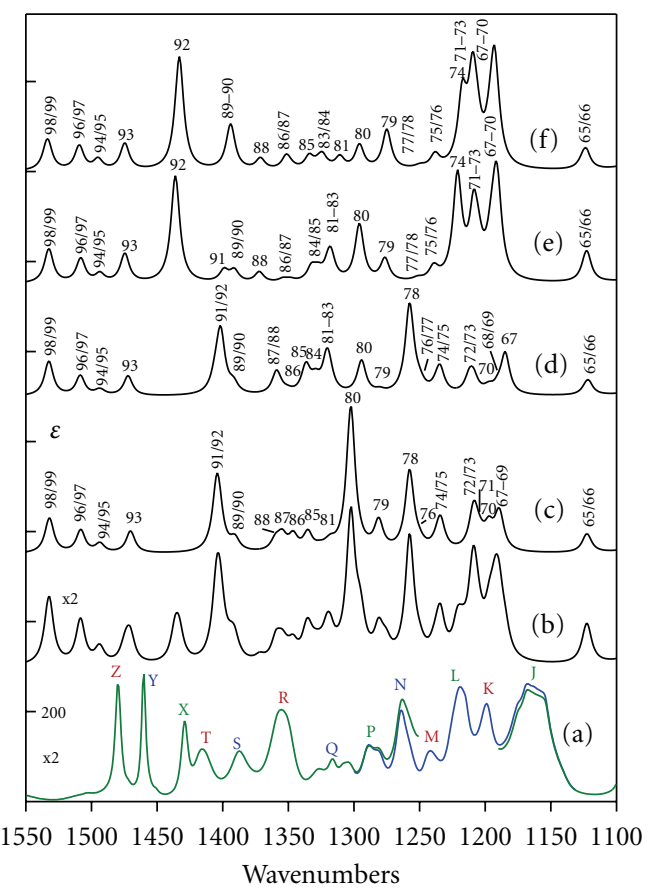

FIgURE 6: Mid-IR (1100-1550 $\mathrm{cm}^{-1}$ ) absorption spectrum of 1. (a) Experimental spectrum (from Figure 3); (b) composite calculated spectrum; (c) B3PW91/6-31G* spectrum of I; (d) B3PW91/6-31G* spectrum of II; (e) B3PW91/6-31G* spectrum of III; (f) B3PW91/6$31 \mathrm{G}^{*}$ spectrum of IV. The composite spectrum is obtained using the percentage populations of I-IV in Table 2. Fundamentals are numbered. Band shapes are Lorentzian $\left(\gamma=4.0 \mathrm{~cm}^{-1}\right)$.

experimental spectra. In particular, the agreement is better than when the experimental spectra are compared to the calculated spectra for conformation I alone. That is, without including the contributions of conformations II, III, and IV, the experimental spectra are much less satisfactorily assigned. On one hand, more than one region clearly demand the presence of II, III, and IV to be convincingly assigned, in particular, the absorption and VCD of the former originate in III, and IV, whose modes 92 lie within broad windows in the spectra of I and II. The absorption and VCD of the latter are not fully resolved. However, in absorption the contributions of III and IV are clearly evident in the high frequency shoulder to the main absorption. In VCD the relative intensities of the two features require the contribution of II. On the other hand, in order to evaluate the alteration in conformational contributions between the two classifications (electronic energies and the free energies cases), an accurate spectral analysis is necessary. We should then deconvolute the experimental spectra taking into account all the predicted bands with an accurate weight. This is the only way how to deduce the true population ratios of the four conformations. However, according to the present analysis, the conformation II contribution appears to be more significant than that of conformation IV.

The analysis of the mid-IR absorption and VCD spectra thus strongly supports the reliability of the predictions of our DFT/6-31G* calculations. In particular, predicted

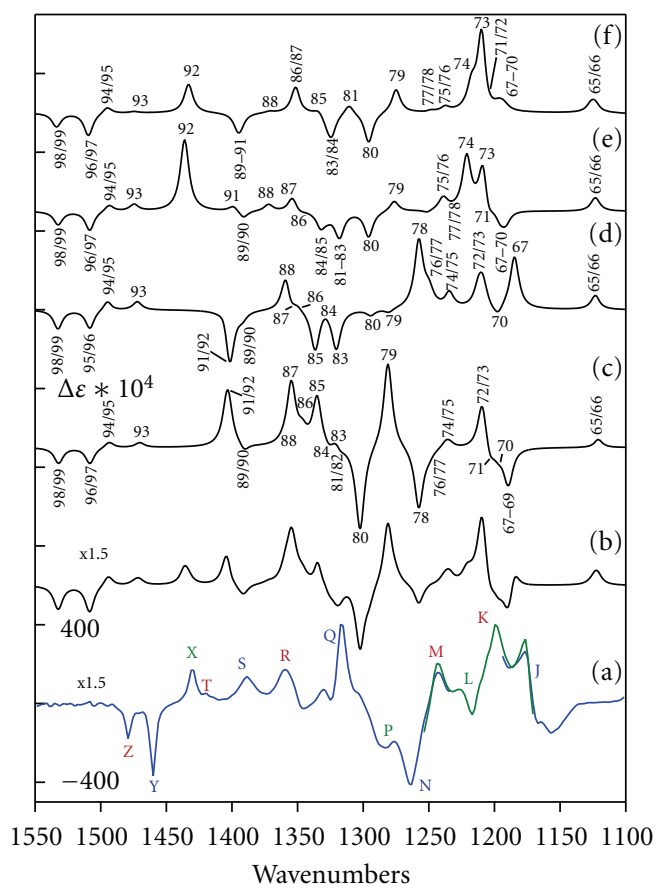

Figure 7: Mid-IR (1100-1550 $\mathrm{cm}^{-1}$ ) VCD spectrum of 1. (a) Experimental spectrum (from Figure 3); (b) composite calculated spectrum; (c) B3PW91/6-31G* spectrum of I; (d) B3PW91/6-31G* spectrum of II; (e) B3PW91/6-31G* spectrum of III; (f) B3PW91/6$31 \mathrm{G}^{*}$ spectrum of IV. The composite spectrum is obtained using the percentage populations of I-IV in Table 2. Fundamentals are numbered. Band shapes are Lorentzian $\left(\gamma=4.0 \mathrm{~cm}^{-1}\right)$.

energy ordering and differences of conformations I-IV are clearly qualitatively correct. Our analysis does not lead to quantitative values for the free energy differences of the four conformations. We have shown previously (in the case of 3methylcyclohexanone [37]) that quantitative deconvolution of the experimental spectra into the component contributions of individual conformations provides free energy differences. However, the greater complexity of a similar analysis of the spectra of $\mathbf{1}$, possessing more conformations and a greater vibrational density of states, has led us to defer such analysis to a future date.

\section{Conclusion}

DFT structures and relative energies of all possible conformations using vibrational spectroscopy of $\mathbf{1}$ (IndaBOX) have been evaluated. Vibrational spectra provide a basis for the conformational analysis. For chiral molecules both IR and VCD spectra can be measured. Calculated IR and VCD spectra of stable conformations are found significantly different. The variation is noticeably greater in the VCD spectra. The limitations to the B3PW91 harmonic calculations at the 6$31 \mathrm{G}^{*}$ basis set level provide a result in credible agreement with the experiment. In particular, without including the contributions of at least first less stable conformations, experimental spectra are much less satisfactorily assigned. As a result, the collective use of IR and VCD spectra is 


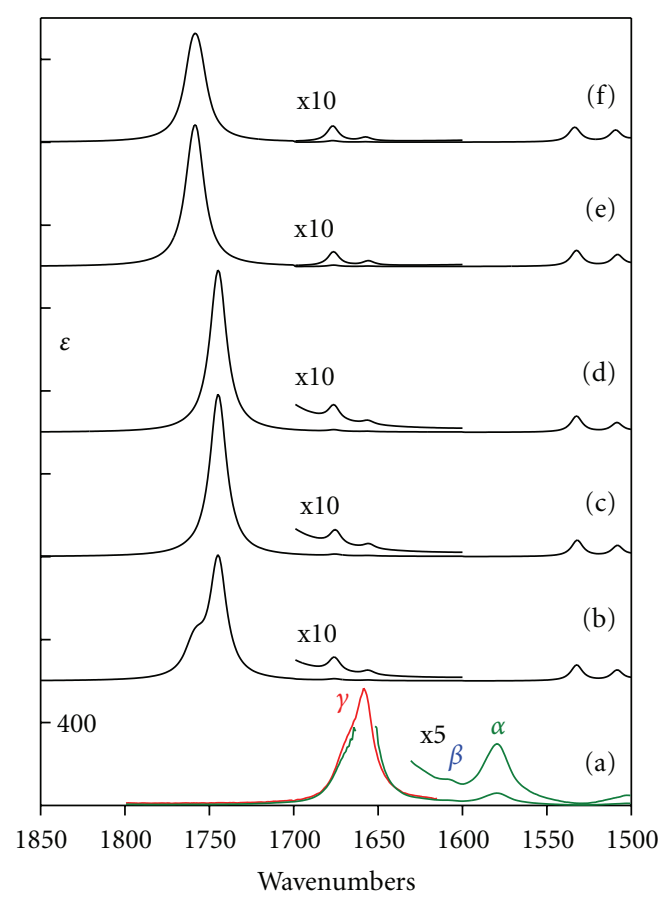

Figure 8: Mid-IR (1500-1850 $\mathrm{cm}^{-1}$ ) absorption spectrum of 1. (a) Experimental spectrum (from Figure 3); (b) composite calculated spectrum; (c) B3PW91/6-31G* spectrum of I; (d) B3PW91/6-31G* spectrum of II; (e) B3PW91/6-31G* spectrum of III; (f) B3PW91/6$31 \mathrm{G}^{*}$ spectrum of IV. The composite spectrum is obtained using the percentage populations of I-IV in Table 2. Fundamentals are numbered. Band shapes are Lorentzian $\left(\gamma=4.0 \mathrm{~cm}^{-1}\right)$.

substantially more powerful than the use of IR spectra alone. Elsewhere the inclusion of other theoretical treatments such as anharmonicity or solvent effect seems unnecessary for the present investigation.

This kind of methodology provides a practical demonstration of VCD profit for full structural analysis. The gain of this direct and rapid tool resides in not requiring chiral reference molecules, single crystal growing approaches, or heavy atom association involved in other techniques. Recently similar studies are in agreement with this conclusion [5457]. The growing use of this technique is also perceived elementary in case of the biological building blocks in order to elucidate proteins conformations and chirality [58]. At the present time, the expanding implementation in other program packages (CADPAC, DALTON, etc.) and the technical transformation of many spectrometers (JASCO, BRUKER, etc.) will certainly empower the VCD utilization.

The development of this strategy relies on the evolution of three related domain. First, the instrumental aspect requires the use of sophisticated materials and electronics to eliminate any external artifact particularly when using a single enantiomer in small quantities. Second, to circumvent the limitations of molecular size, the theoretical methods need the implementation of more procedures. Third, the computational technology should optimize more efficiently the use of hardware resources. These hints will provide an attractive alternative to other techniques for both absolute

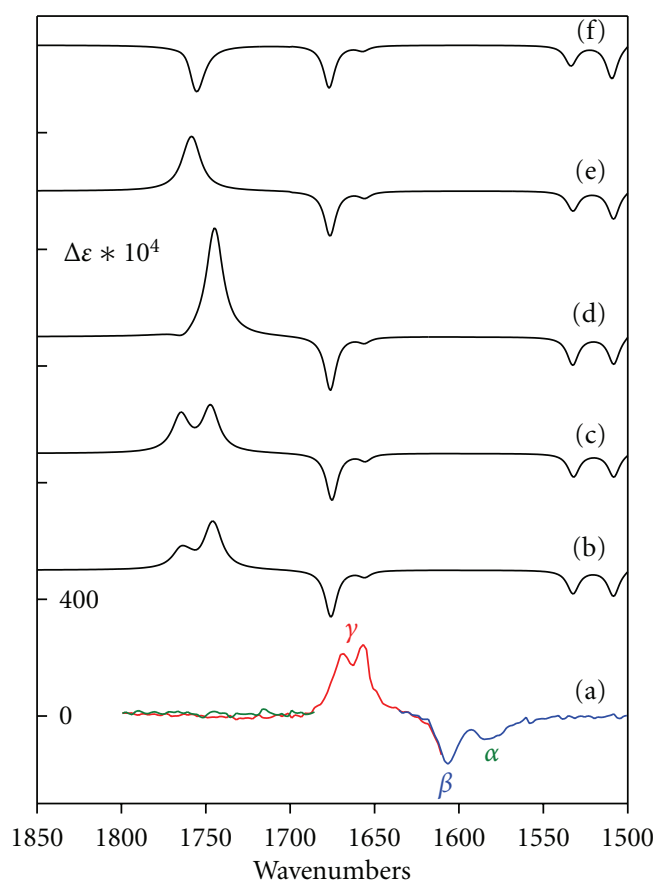

Figure 9: Mid-IR (1500-1850 $\mathrm{cm}^{-1}$ ) VCD spectrum of 1. (a) Experimental spectrum (from Figure 3); (b) composite calculated spectrum; (c) B3PW91/6-31G* spectrum of I; (d) B3PW91/6-31G* spectrum of II; (e) B3PW91/6-31G* spectrum of III; (f) B3PW91/6$31 \mathrm{G}^{*}$ spectrum of IV. The composite spectrum is obtained using the percentage populations of I-IV in Table 2. Fundamentals are numbered. Band shapes are Lorentzian $\left(\gamma=4.0 \mathrm{~cm}^{-1}\right)$.

configuration determination and conformational analysis. Perhaps this will persuade more companies, notably pharmaceutical, to take advantage from this promising tool.

\section{Acknowledgments}

The authors are grateful to F. J. Devlin (Department of Chemistry, University of Southern California, Los Angeles) for discussions and assistance endowed to this work. This paper is dedicated to 70th anniversary of Professor Philip J. Stephens (specially for his contribution to the VCD spectroscopy).

\section{References}

[1] J. W. Faller and A. Lavoie, "Highly enantioselective DielsAlder catalysis with a chiral ruthenium bisoxazoline complex," Journal of Organometallic Chemistry, vol. 630, no. 1, pp. 17-22, 2001.

[2] D. Rechavi, B. Albela, L. Bonneviot, and M. Lemaire, "Understanding the enantioselectivity of a heterogeneous catalyst: the influence of ligand loading and of silica passivation," Tetrahedron, vol. 61, no. 29, pp. 6976-6981, 2005.

[3] S. Tanaka, M. Tada, and Y. Iwasawa, "Enantioselectivity promotion by achiral surface functionalization on $\mathrm{SiO} 2-$ supported Cu-bis(oxazoline) catalysts for asymmetric DielsAlder reactions," Journal of Catalysis, vol. 245, no. 1, pp. 173183, 2007. 
[4] J.-M. Lee, J. Kim, Y. Shin et al., "Heterogeneous asymmetric Henry reaction using a chiral bis(oxazoline)-copper complex immobilized on magnetically separable mesocellular mesoporous silica support," Tetrahedron Asymmetry, vol. 21, no. 3, pp. 285-291, 2010.

[5] E. Framery, B. Andrioletti, and M. Lemaire, "Recent progress in homogeneous supported asymmetric catalysis: example of the BINAP and the BOX ligands," Tetrahedron Asymmetry, vol. 21, no. 9-10, pp. 1110-1124, 2010.

[6] A. S. Abu-Surrah, M. Kettunen, K. Lappalainen, U. Piironen, M. Klinga, and M. Leskelä, "Synthesis of new chiral diimine palladium(II) and nickel(II) complexes bearing oxazolineand myrtanyl-based nitrogen ligands. Crystal structure of the C2-symmetric complex [(1R,2S)-inda-boxPdCl2]," Polyhedron, vol. 21, no. 1, pp. 27-31, 2002.

[7] K. Matsumoto, K. Jitsukawa, and H. Masuda, "Preparation of new bis(oxazoline) ligand bearing non-covalent interaction sites and an application in the highly asymmetric Diels-Alder reaction," Tetrahedron Letters, vol. 46, no. 34, pp. 5687-5690, 2005.

[8] D. Frain, F. Kirby, P. McArdle, and P. O’Leary, "Preparation, structure and catalytic activity of copper(II) complexes of novel 4,4'-BOX ligands," Tetrahedron Letters, vol. 51, no. 31, pp. 4103-4106, 2010.

[9] S. Telalović and U. Hanefeld, "Noncovalent immobilization of chiral cyclopropanation catalysts on mesoporous TUD1: comparison of liquid-phase and gas-phase ion-exchange," Applied Catalysis A, vol. 372, no. 2, pp. 217-223, 2010.

[10] N. Merle, K. W. Törnroos, V. R. Jensen, and E. Le Roux, "Influence of multidentate N-donor ligands on highly electrophilic zinc initiator for the ring-opening polymerization of epoxides," Journal of Organometallic Chemistry, vol. 696, no. 8, pp. 1691-1697, 2011.

[11] P. Fulde, in Electron Correlations in Molecules and Solids, p. 39, Spinger, Berlin, Germany, 1995.

[12] R. M. Dreizler and E. K. U. Gross, Density Functional Theory, Springer, Berlin, Germany, 1990.

[13] E. Charney, The Molecular Basis of Optical Activity: Optical Rotatory Dispersion and Circular Dichroism, Wiley, New York, NY, USA, 1979.

[14] L. D. Barron, Molecular Light Scattering and Optical Activity, Cambridge University Press, Cambridge, UK, 1982.

[15] T. M. Lwory, Optical Rotatory Power, Longmans, Green and Co, London, UK, 1935.

[16] P. J. Stephens, "The theory of vibrational circular dichroism," in Encyclopedia of Spectroscopy and Spectrometry, p. 2415, Academic Press, London, UK, 1999.

[17] R. W. Kawiecki, F. Devlin, P. J. Stephens, R. D. Amos, and N. C. Handy, "Vibrational circular dichroism of propylene oxide," Chemical Physics Letters, vol. 145, no. 5, pp. 411-417, 1988.

[18] L. Rosenfeld, "Quantenmechanische theorie der natürlichen optischen aktivität von flüssigkeiten und gasen," Zeitschrift für Physik, vol. 52, no. 3-4, pp. 161-174, 1929.

[19] P. J. Stephens, "Gauge dependence of vibrational magnetic dipole transition moments and rotational strengths," Journal of Physical Chemistry, vol. 91, no. 7, pp. 1712-1715, 1987.

[20] M. A. Lowe, G. A. Segal, and P. J. Stephens, "The theory of vibrational circular dichroism: trans-1,2dideuteriocyclopropane," Journal of the American Chemical Society, vol. 108, no. 2, pp. 248-256, 1986.

[21] P. J. Stephens, "Theory of vibrational circular dichroism," Journal of Physical Chemistry, vol. 89, no. 5, pp. 748-752, 1985.
[22] L. A. Nafie and T. B. Freedman, "Vibronic coupling theory of infrared vibrational transitions," The Journal of Chemical Physics, vol. 78, no. 12, pp. 7108-7116, 1982.

[23] R. G. Parr and W. Yang, Density-Functional Theory of Atoms and Molecules, Oxford University Press, New York, NY, USA, 1989.

[24] A. D. Becke, "Density-functional thermochemistry. III. The role of exact exchange," The Journal of Chemical Physics, vol. 98, no. 7, pp. 5648-5652, 1993.

[25] J. P. Perdew and W. Yue, "Accurate and simple density functional for the electronic exchange energy: generalized gradient approximation," Physical Review B, vol. 33, no. 12, pp. 8800-8802, 1986.

[26] W. J. Hehre, L. Radom, P. V. R. Schleyer, and J. A. Pople, Ab Initio Molecular Orbital Theory, John Wiley \& Sons, New York, NY, USA, 1986.

[27] GAUSSIAN, M. J. Frisch, Gaussian, Inc., Pittsburgh, Pa, USA, 1998.

[28] F. J. Devlin, P. J. Stephens, J. R. Cheeseman, and M. J. Frisch, "Prediction of vibrational circular dichroism spectra using density functional theory: camphor and fenchone," Journal of the American Chemical Society, vol. 118, no. 26, pp. 6327-6328, 1996.

[29] F. J. Devlin, P. J. Stephens, J. R. Cheeseman, and M. J. Frisch, "Ab initio prediction of vibrational absorption and circular dichroism spectra of chiral natural products using density functional theory: camphor and fenchone," Journal of Physical Chemistry A, vol. 101, no. 35, pp. 6322-6333, 1997.

[30] F. J. Devlin, P. J. Stephens, J. R. Cheeseman, and M. J. Frisch, "Ab initio prediction of vibrational absorption and circular dichroism spectra of chiral natural products using density functional theory: $\alpha$-Pinene," Journal of Physical Chemistry A, vol. 101, no. 51, pp. 9912-9924, 1997.

[31] C. S. Ashvar, P. J. Stephens, T. Eggimann, and H. Wieser, "Vibrational circular dichroism spectroscopy of chiral pheromones: frontalin (1,5-dimethyl-6,8-dioxabicyclo [3.2.1] octane)," Tetrahedron Asymmetry, vol. 9, no. 7, pp. 1107-1110, 1998.

[32] C. S. Ashvar, F. J. Devlin, P. J. Stephens, K. L. Bak, T. Eggimann, and H. Wieser, "Vibrational absorption and circular dichroism of mono- and dimethyl derivatives of 6,8dioxabicyclo[3.2.1] octane," Journal of Physical Chemistry A, vol. 102, no. 34, pp. 6842-6857, 1998.

[33] C. S. Ashvar, F. J. Devlin, and P. J. Stephens, "Molecular structure in solution: an ab initio vibrational spectroscopy study of phenyloxirane," Journal of the American Chemical Society, vol. 121, no. 12, pp. 2836-2849, 1999.

[34] A. Aamouche, F. J. Devlin, and P. J. Stephens, "Determination of absolute configuration using circular dichroism: troger's Base revisited using vibrational circular dichroism," Chemical Communications, no. 4, pp. 361-362, 1999.

[35] A. Aamouche, F. J. Devlin, and P. J. Stephens, "Structure, vibrational absorption and circular dichroism spectra, and absolute configuration of Tröger's base," Journal of the American Chemical Society, vol. 122, no. 10, pp. 2346-2354, 2000.

[36] F. J. Devlin and P. J. Stephens, "Ab initio density functional theory study of the structure and vibrational spectra of cyclohexanone and its isotopomers," Journal of Physical Chemistry A, vol. 103, no. 4, pp. 527-538, 1999.

[37] F. J. Devlin and P. J. Stephens, "Conformational analysis using ab initio vibrational spectroscopy: 3- Methylcyclohexanone," Journal of the American Chemical Society, vol. 121, no. 32, pp. 7413-7414, 1999. 
[38] P. J. Stephens and F. J. Devlin, "Determination of the structure of chiral molecules using ab initio vibrational circular dichroism spectroscopy," Chirality, vol. 12, no. 4, pp. 172-179, 2000.

[39] A. Aamouche, F. J. Devlin, and P. J. Stephens, "Conformations of chiral molecules in solution: ab initio vibrational absorption and circular dichroism studies of 4,4a,5,6,7,8-hexahydro4a-methyl-2(3H)-naphthalenone and 3,4,8,8a-tetrahydro-8amethyl-1,6(2H,7H)-naphthalenedione," Journal of the American Chemical Society, vol. 122, no. 30, pp. 7358-7367, 2000.

[40] A. Aamouche, F. J. Devlin, P. J. Stephens, J. Drabowicz, B. Bujnicki, and M. Mikołajczyk, "Vibrational circular dichroism and absolute configuration of chiral sulfoxides: tert-butyl methyl sulfoxide," Chemistry European Journal, vol. 6, no. 24, pp. 4479-4486, 2000.

[41] P. J. Stephens, A. Aamouche, F. J. Devlin, S. Superchi, M. I. Donnoli, and C. Rosini, "Determination of absolute configuration using vibrational circular dichroism spectroscopy: the chiral sulfoxide 1-(2-methylnaphthyl) methyl sulfoxide," Journal of Organic Chemistry, vol. 66, no. 11, pp. 3671-3677, 2001.

[42] F. J. Devlin, P. J. Stephens, P. Scafato, S. Superchi, and C. Rosini, "Determination of absolute configuration using vibrational circular dichroism spectroscopy: the chiral sulfoxide 1thiochroman S-oxide," Tetrahedron Asymmetry, vol. 12, no. 11, pp. 1551-1558, 2001.

[43] P. J. Stephens, F. J. Devlin, and A. Aamouche, "Determination of the structures of chiral molecules using Vibrational Circular Dichroism spectroscopy," in Chirality: Physical Chemistry, J. M. Hicks, Ed., vol. 810 of ACS Symposium Series, chapter 2, pp. 18-33, 2002.

[44] P. J. Stephens, F. J. Devlin, and J.-J. Pan, “The determination of the absolute configurations of chiral molecules using Vibrational Circular Dichroism (VCD) spectroscopy," Chirality, vol. 20, no. 5, pp. 643-663, 2008.

[45] Company internal report, Sanofi-Aventis, Toulouse, France, 1999.

[46] E. Da Plama Carreiro, S. Chercheja, A. J. Burke, J. P.P. Ramalho, and A. I. Rodrigues, "Isbut-box: a new chiral C2 symmetric bis-oxazoline for catalytic enantioselective synthesis," Journal of Molecular Catalysis A, vol. 236, no. 1-2, pp. 38-45, 2005.

[47] R. P. Singh, "Spectroscopic studies on complexes of magnesium (II) with C2-chiral bis-oxazolines," Spectrochimica Acta Part A, vol. 53, no. 11, pp. 1713-1717, 1997.

[48] M. P. Sibi, G. Petrovic, and J. Zimmerman, "Enantioselective radical addition/trapping reactions with $\alpha, \beta$-disubstituted unsaturated imides. Synthesis of anti-propionate aldols," Journal of the American Chemical Society, vol. 127, no. 8, pp. 2390-2391, 2005.

[49] G. Desimoni, G. Faita, and K. A. Jørgensen, "C2-symmetric chiral bis(oxazoline) ligands in asymmetric catalysis," Chemical Reviews, vol. 106, no. 9, pp. 3561-3651, 2006.

[50] I. Gallou and C. H. Senanayake, "cis-1-amino-2-indanol in drug design and applications to asymmetric processes," Chemical Reviews, vol. 106, no. 7, pp. 2843-2874, 2006.

[51] T. Tsubogo, S. Saito, K. Seki, Y. Yamashita, and S. Kobayashi, "Development of catalytic asymmetric 1,4-addition and [3+ 2] cycloaddition reactions using chiral calcium complexes," Journal of the American Chemical Society, vol. 130, no. 40, pp. 13321-13332, 2008.

[52] K. Lang, J. Park, and S. Hong, "Development of bifunctional aza-bis(oxazoline) copper catalysts for enantioselective henry reaction," Journal of Organic Chemistry, vol. 75, no. 19, pp. 6424-6435, 2010.
[53] A. K. Ghosh, "Capturing the essence of organic synthesis: from bioactive natural products to designed molecules in today's medicine," Journal of Organic Chemistry, vol. 75, no. 23, pp. 7967-7989, 2010.

[54] T. Bürgi, A. Vargas, and A. Baiker, "VCD spectroscopy of chiral cinchona modifiers used in heterogeneous enantioselective hydrogenation: conformation and binding of non-chiral acids," Journal of the Chemical Society, Perkin Transactions, vol. 2, no. 9, pp. 1596-1601, 2002.

[55] J. Vachon, S. Harthong, B. Dubessy et al., "The absolute configuration of an inherently chiral phosphonatocavitand and its use toward the enantioselective recognition of Ladrenaline," Tetrahedron Asymmetry, vol. 21, no. 11-12, pp. 1534-1541, 2010.

[56] H. Izumi, S. Futamura, L. A. Nafie, and R. K. Dukor, "Determination of molecular stereochemistry using vibrational circular dichroism spectroscopy: Absolute configuration and solution conformation of 5-formyl-cis,cis-1,3,5-trimethyl-3hydroxymethylcyclohexane-1-carboxylic acid lactone," Chemical Record, vol. 3, no. 2, pp. 112-119, 2003.

[57] F. J. Devlin, P. J. Stephens, and P. Besse, "Are the absolute configurations of 2-(1-hydroxyethyl)-chromen-4-one and its 6-bromo derivative determined by X-ray crystallography correct? A vibrational circular dichroism study of their acetate derivatives," Tetrahedron Asymmetry, vol. 16, no. 8, pp. 15571566, 2005.

[58] T. A. Keiderling, "Protein and peptide secondary structure and conformational determination with vibrational circular dichroism," Current Opinion in Chemical Biology, vol. 6, no. 5, pp. 682-688, 2002. 


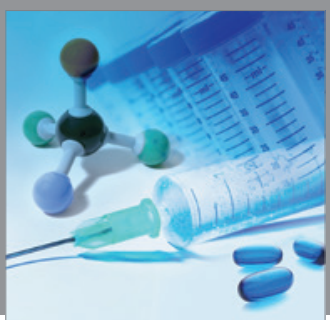

International Journal of

Medicinal Chemistry

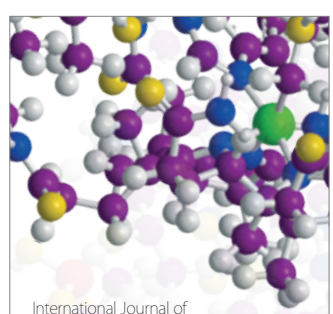

Carbohydrate Chemistry

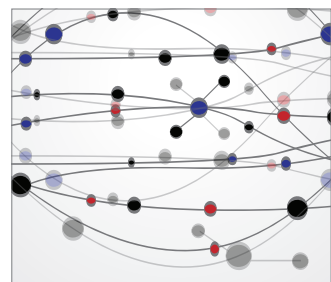

The Scientific World Journal
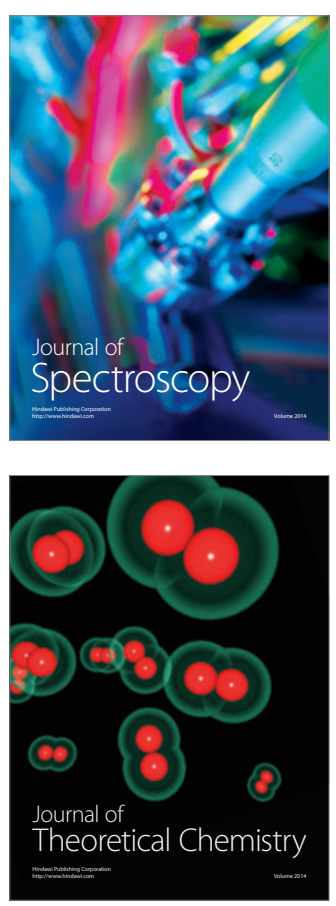
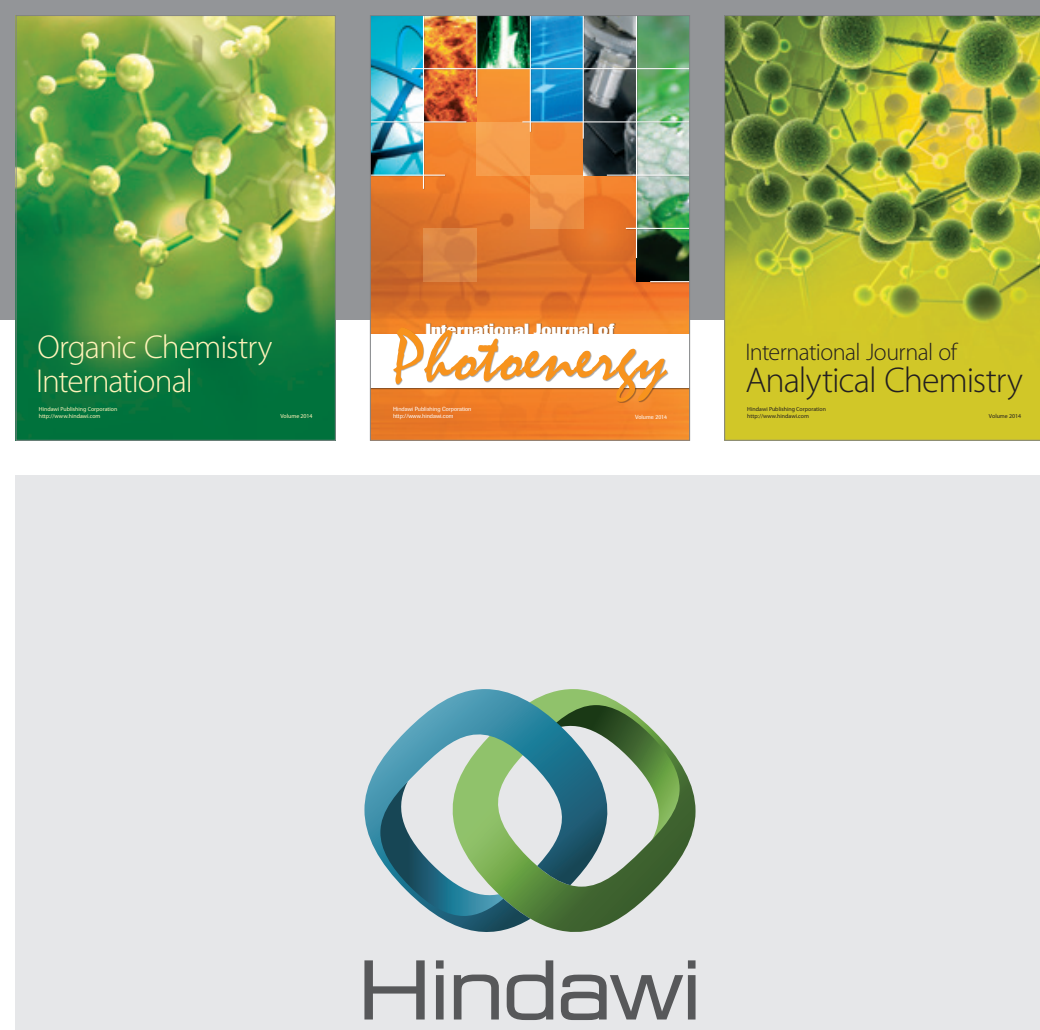

Submit your manuscripts at

http://www.hindawi.com
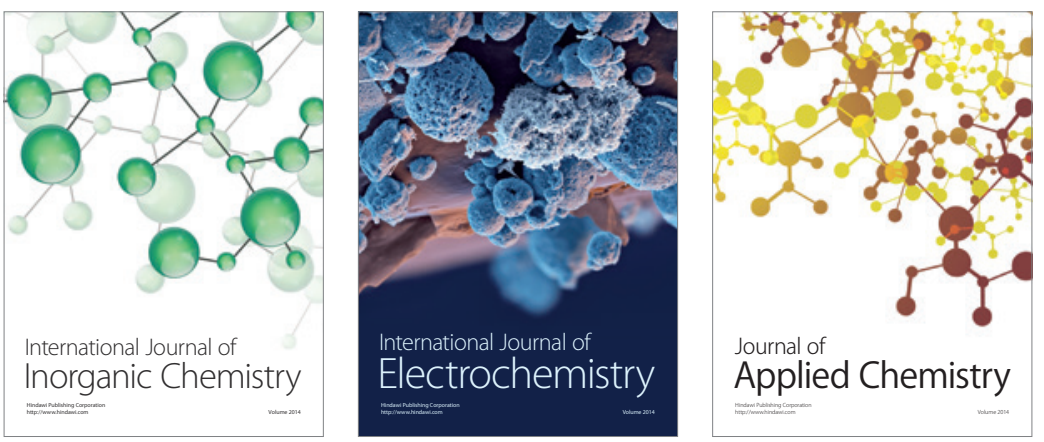

Journal of

Applied Chemistry
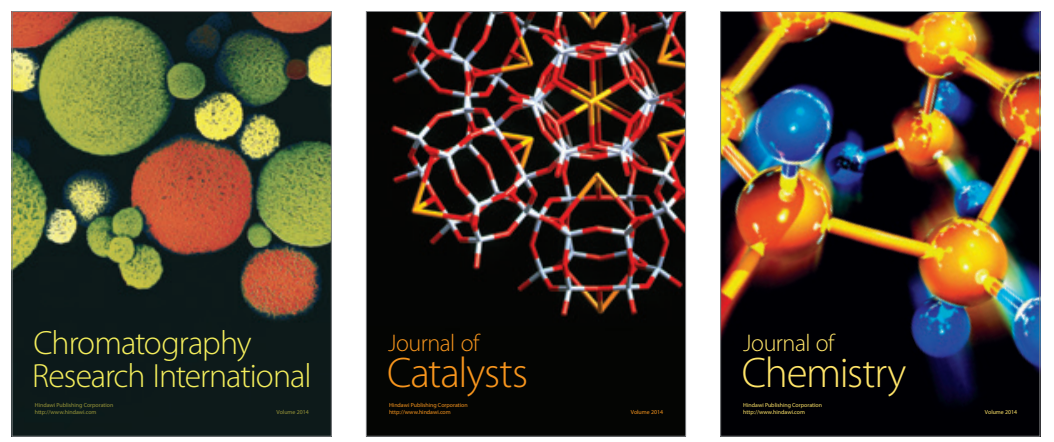
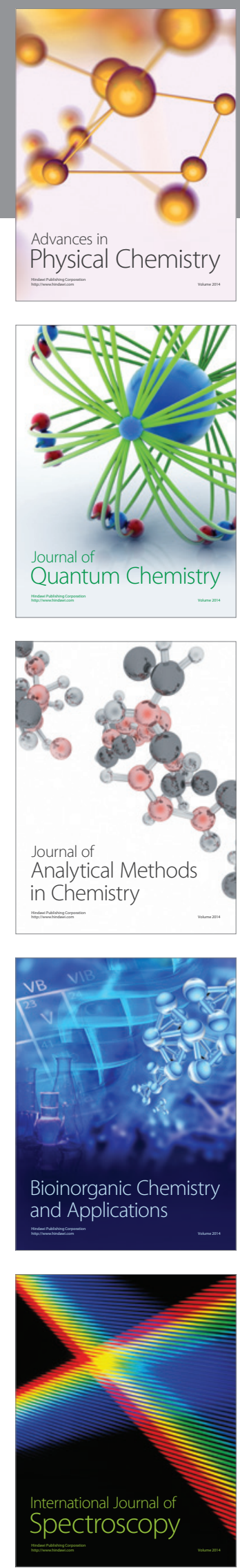